\title{
Cytogenetics of Mimosa bimucronata (DC.) O. Kuntze (Mimosoideae, Leguminosae): chromosome number, polysomaty and meiosis
}

\author{
Denise Olkoski ${ }^{1}$ and Maria Teresa Schifino Wittmann ${ }^{1 *}$
}

Received 15 April 2010

Accepted 27 June 2010

\begin{abstract}
Chromosome numbers (somatic and/or gametic) were determined in 50 populations of M. bimucronata (DC.) $O$. Kuntze collected in the species area of distribution in Rio Grande do Sul, south Brazil. All populations were diploid $(2 n=2 x=26$, $n=13$ ). Polysomatic (mostly tetraploid) cells were detected in the seedlings root-tip cells in 39 out of the 41 populations examined, ranging from 3.0 to $28.2 \%$ among populations, but were absent in the root-tips of grown plants. Polysomaty was as well absent in pollen-mother cells. In M. bimucronata pollen-mother cells are joined two-by-two before the onset of meiosis, remaining attached during all the meiotic division until the formation of pollen grain polyads, composed of two sets of four pollen grains each, that are dispersed in this way, which, according to previous suggestions would be an adaptation to ensure high seed set after a single pollination event.
\end{abstract}

Key words: “maricá”, intraspecific variability, polyads.

\section{INTRODUCTION}

The genus Mimosa L. (Mimosoideae, Leguminosae) comprises around 530 species, most of them native to the Americas (Barneby 1991). Many of Mimosa species are multipurpose trees, and among them is M. bimucronata (DC.) O. Kuntze, which occurs in Paraguay, Argentina and Brazil (from the State of Alagoas in the northeast to the State of Rio Grande do Sul in the south). Two varieties of the species are described, M. bimucronata (DC.) O. Kuntze var. bimucronata, the most widespread variety, and M. bimucronata var. adenocarpa Hasll, restricted to Paraguay, which differs only by the more abundant trichomas in M bimucronata var. adenocarpa.

M. bimucronata, popularly known in Brazil as "marica", "silva", "espinheiro" or "angiquinho" (Backes and Irgang 2002) is widely used as living and defensive fences (due to its thorns), for medicinal purposes and for honey production and its wood is employed in building, carpentry and as fuel (Burkart 1979, Barneby 1991, Lorenzi 1998). Many authors refer to the species importance in the recovery of degraded lands (Reitz et al. 1983, Marchiori 1993, Bernaci et al. 1998, Nascimento et al. 2003, Barbosa and Faria 2006, Bitencourt et al. 2007).

The basic chromosome number in genus Mimosa is suggested as being $x=13$, probably derived by dysploidy from the more common $\mathrm{x}=14$ in the Mimosoideae (Goldblatt 1981). Most of Mimosa species studied (around $10 \%$ of the genus) are diploid, with $2 \mathrm{n}=2 \mathrm{x}=26$, others are tetraploid $(2 \mathrm{n}=4 \mathrm{x}=52)$ and a few are octaploid $(2 \mathrm{n}=8 \mathrm{x}=$ 104) (Federov 1969, Alvez and Custódio 1989, Seijo 1993, 1999, 2000, Seijo and Fernández 2001, Goldblatt and Johnson 2009). Intraspecific variability (generally diploid and tetraploid cytotypes) have been reported in M. pudica

\footnotetext{
${ }^{1}$ Universidade Federal do Rio Grande do Sul, Faculdade de Agronomia, Departamento de Plantas Forrageiras e Agrometeorologia, CP 15100, 91.501-970, Porto Alegre, RS, Brazil.*E-mail: mtschif@ufrgs.br
} 
L. (Goldblatt and Johnson 2009), M. somnians Humb. \& Bonpl. ex Willd. (Seijo 1993, 2000), M. balansae Micheli (Seijo and Fernández 2001), M. debilis Humb. \& Bonpl. ex Willd.,and M. nuda Benth (Morales et al. 2010). It should be stressed that most chromosome counts are restricted to one or fewer populations, therefore intraspecific variability may be more common than reported. For M. bimucronata, Seijo $(1999,2000)$ reported $2 n=26$ for populations from Argentina and Paraguay.

Polysomaty in root tip meristematic cells, the presence of cells with higher ploidy levels, have been reported in some Mimosa species by Witkus and Berger (1947) and Seijo (1993, 1999). According to Witkus and Berger (1947) polysomaty could be a way to assure a quick seedling establishment.

Seijo and Neffa (2004) described the formation of polyads, compound pollen grains, in $M$. bimucronata. Compound pollen grains are found in ca. $15 \%$ of the angiosperm families (Kenrick and Knox 1982) and would be a mechanism to reinforce pollination efficiency.

The aim of this paper was to analyze chromosome numbers and meiotic behaviour in M. bimucronata populations from the species distribution area in the State of Rio Grande do Sul, South Brazil, to study frequency and distribution of polysomaty.

\section{MATERIAL AND METHODS}

All populations analyzed were collected (fruits with mature seeds and/or inflorescences) along the area of distribution of the species in the State of Rio Grande do Sul during the years of 2008 and 2009. Taxonomic vouchers are deposited at the ICN Herbarium (Departamento de Botânica, Instituto de Biociências, Universidade Federal do Rio Grande do Sul, Porto Alegre, Brazil) (Table 1).

For somatic chromosome number determination, the seeds were scarified with sand paper or by a small cut in the testa and germinated in petri dishes lined with moist filter paper. Following Dahmer et al. (2009) with some modifications, root-tips around $1 \mathrm{~cm}$ length were pretreated with a saturated solution of paradichlorobenzene for $24 \mathrm{~h}$ at $4{ }^{\circ} \mathrm{C}$, fixed in 6:3: 1 (ethanol: chloroform: acetic acid) for 12-24 $\mathrm{h}$ and stored in $70 \%$ alcohol below $0{ }^{\circ} \mathrm{C}$ until slide preparation. For that, the roots were hydrolyzed with $1 \mathrm{~N}$ $\mathrm{HCl}$ at $60^{\circ} \mathrm{C}$ for $8-10 \mathrm{~min}$, stained by Feulgen for around 2 $\mathrm{h}$, treated with $2 \%$ pectinase for $2 \mathrm{~min}$, and squashed in $2 \%$ propionic carmine. At least ten cells with good chromosome spreading and with chromosomes in equivalent degree of contraction were analysed per plant. The best cells were photographed and/or registered by a digital image capturing system.

Percentage of polysomatic cells were determined in the same slides used for chromososome number counting. As a cross check to eliminate the possible cause of polysomaty being a result of pretreatment, slides of the same populations were prepared by the same technique as described above, but with no pretreatment. Additionaly, individuals of the five populations with higher frequency of polysomatic cells in meristematic root- tip cells $(4,9,11$, 24 and 37) were grown in pots with commercial garden soil in a greenhouse. After ca. 11 months, root-tips were collected from these plants and went to the same technical procedures (fixation, pretreatment, staining and squashing) as the seedlings root-tips.

For gametic chromosome number determination and meiotic analysis, young inflorescences, collected at the field, were immediately fixed in Carnoy 6: 3: 1 (ethanol: clorophorm: acetic acid) for $24 \mathrm{~h}$ at room temperature and stored in $70 \%$ alcohol below $0{ }^{\circ} \mathrm{C}$ until slide preparation. Slides were prepared by squashing the anthers of one flower bud per slide in $2 \%$ propionic carmine. All available cells in all meiotic stages were analyzed. The best cells were photographed and/or registered by a digital image capturing system.

\section{RESULTS AND DISCUSSION}

This is the first work where a great number of $M$ bimucronata populations (50) were cytogenetically analyzed. Previous works were restricted to a few populations from Paraguay and Argentina (Seijo 1999, 2000).

Somatic chromosome numbers were determined in 193 individuals from 41 populations and gametic chromosome numbers in 49 individuals of 12 populations (Table 1). All individuals were diploid with $2 \mathrm{n}=2 \mathrm{x}=26$ (Figure 1 a to $c$ ) or $n=13$ (Figure $2 b$ ) confirming the diploid nature of $M$. bimucronata, as already reported for two populations of de $M$. bimucronata var. bimucronata from Argentina (Seijo 1999) and for one population of $M$. bimucronata var. adenocarpa from Paraguay (Seijo 2000) No intraspecific variability for chromosome number was detected among the analyzed populations.

Polysomatic cells were detected in the seedlings roottip cells, with and without pretreatment, in all the 41 populations examined except populations 13 and 21 
Table 1. Population, locality collection, voucher number, geographical coordinates, chromosome number and percentage of polysomatic cells in the $M$. bimucronata poupulations analyzed

\begin{tabular}{|c|c|c|c|c|c|}
\hline Population & Locality and voucher & $\begin{array}{c}\text { Geographical } \\
\text { coordinates }\end{array}$ & $n=13^{a}$ & $2 n=26^{a}$ & $\begin{array}{c}\text { Polysomatic } \\
\text { cells }(\%)^{\mathbf{b}}\end{array}$ \\
\hline 1 & $\begin{array}{l}\text { Brazil, Rio Grande do Sul, Porto Alegre. D. } \\
\text { Olkoski } 01 \text { (ICN 162475) }\end{array}$ & $\begin{array}{l}\mathrm{S} 30^{\circ} 04^{\prime} 10.46^{\prime \prime} ; \\
\mathrm{W} 51^{\circ} 08^{\prime} 35.01^{\prime \prime}\end{array}$ & $\begin{array}{c}6 \\
(41)\end{array}$ & $\begin{array}{c}10 \\
(135)\end{array}$ & 9.6 \\
\hline 2 & $\begin{array}{l}\text { Brazil, Rio Grande do Sul, Porto Alegre. D. } \\
\text { Olkoski } 02 \text { (ICN 162476) }\end{array}$ & $\begin{array}{l}\mathrm{S} 30^{\circ} .04^{\prime} 00.74^{\prime \prime} \\
\text { W5 } 1^{\circ} .07^{\prime} 16.09^{\prime \prime}\end{array}$ & $\begin{array}{c}5 \\
(25)\end{array}$ & $\begin{array}{c}10 \\
(64)\end{array}$ & 4.7 \\
\hline 3 & $\begin{array}{l}\text { Brazil, Rio Grande do Sul, Santa Maria. D. } \\
\text { Olkoski } 03 \text { (ICN 162477) }\end{array}$ & 等 & & $\begin{array}{c}9 \\
(115)\end{array}$ & 8.7 \\
\hline 4 & $\begin{array}{l}\text { Brazil, Rio Grande do Sul, Cachoeira do Sul. D. } \\
\text { Olkoski } 04 \text { (ICN 162478) }\end{array}$ & $\begin{array}{l}\mathrm{S} 30^{\circ} 15^{\prime} 49.0^{\prime \prime} \\
\mathrm{W} 52^{\circ} 50^{\prime} 48.5^{\prime \prime}\end{array}$ & & $\begin{array}{c}10 \\
(97)\end{array}$ & 10.30 \\
\hline 5 & $\begin{array}{l}\text { Brazil, Rio Grande do Sul, Restinga Seca. D. } \\
\text { Olkoski } 05 \text { (ICN 162479) }\end{array}$ & $\begin{array}{l}\text { S294'14.48”; } \\
\text { W53 } .21^{\prime} 48.07^{\prime \prime}\end{array}$ & & $\begin{array}{c}4 \\
(43)\end{array}$ & 18.6 \\
\hline 6 & $\begin{array}{l}\text { Brazil, Rio Grande do Sul, Viamão. D. Olkoski } \\
06 \text { (ICN 162480) }\end{array}$ & $\begin{array}{l}\text { S } 30^{\circ} 08^{\prime} 05.5^{\prime \prime} \\
\text { W } 50^{\circ} 55^{\prime} 06.4^{\prime \prime}\end{array}$ & & $\begin{array}{c}9 \\
(116)\end{array}$ & 7.7 \\
\hline 7 & $\begin{array}{l}\text { Brazil, Rio Grande do Sul, Viamão. D. Olkoski } \\
07 \text { (ICN 162481) }\end{array}$ & $\begin{array}{l}\text { S } 30^{\circ} 08^{\prime} 05.7^{\prime \prime} \\
\text { W } 50^{\circ} 55^{\prime} 06.6^{\prime \prime}\end{array}$ & & $\begin{array}{c}3 \\
(27)\end{array}$ & 3.7 \\
\hline 8 & $\begin{array}{l}\text { Brazil, Rio Grande do Sul, Viamão. D. Olkoski } \\
08 \text { (ICN 162482) }\end{array}$ & $\begin{array}{l}\text { S } 30^{\circ} 05^{\prime} 35.1 " \\
\text { W } 50^{\circ} 59^{\prime} 52.1^{\prime \prime}\end{array}$ & & $\begin{array}{c}7 \\
(65)\end{array}$ & 6.1 \\
\hline 9 & $\begin{array}{l}\text { Brazil, Rio Grande do Sul, Porto Alegre. D. } \\
\text { Olkoski } 09 \text { (ICN162483) }\end{array}$ & $\begin{array}{l}\text { S } 30^{\circ} 00^{\prime} 51.7^{\prime \prime} \\
\text { W } 51^{\circ} 19^{\prime} 02.2^{\prime \prime}\end{array}$ & & $\begin{array}{c}3 \\
(39)\end{array}$ & 28.2 \\
\hline 10 & $\begin{array}{l}\text { Brazil, Rio Grande do Sul, Eldourado do Sul. D. } \\
\text { Olkoski } 010 \text { (ICN162484) }\end{array}$ & $\begin{array}{l}\text { S } 30^{\circ} 03^{\prime} 09.7^{\prime \prime} \\
\text { W } 51^{\circ} 25^{\prime} 59.7^{\prime \prime}\end{array}$ & & $\begin{array}{c}6 \\
(62)\end{array}$ & 16.1 \\
\hline 11 & $\begin{array}{l}\text { Brazil, Rio Grande do Sul, Eldourado do Sul. D. } \\
\text { Olkoski } 011 \text { (ICN162485) }\end{array}$ & $\begin{array}{l}\text { S } 30^{\circ} 04^{\prime} 47.4^{\prime \prime} \\
\text { W } 51^{\circ} 36^{\prime} 13.1^{\prime \prime}\end{array}$ & & $\begin{array}{c}7 \\
(143)\end{array}$ & 31.5 \\
\hline 12 & $\begin{array}{l}\text { Brazil, Rio Grande do Sul, Arroio dos Ratos. D. } \\
\text { Olkoski } 012 \text { (ICN162486) }\end{array}$ & $\begin{array}{l}\text { S } 30^{\circ} 06^{\prime} 25.1^{\prime \prime} \\
\text { W } 51^{\circ} 45^{\prime} 15.2^{\prime \prime}\end{array}$ & & $\begin{array}{c}5 \\
(50)\end{array}$ & 16.0 \\
\hline 13 & $\begin{array}{l}\text { Brazil, Rio Grande do Sul, Butiá. D. Olkoski } 013 \\
\text { (ICN162487) }\end{array}$ & $\begin{array}{l}\text { S } 30^{\circ} 07^{\prime} 24.9^{\prime \prime} \\
\text { W } 51^{\circ} 53^{\prime} 35.4^{\prime \prime}\end{array}$ & & $\begin{array}{c}3 \\
(25)\end{array}$ & 0.0 \\
\hline 14 & $\begin{array}{l}\text { Brazil, Rio Grande do Sul, Minas do Leão. } \\
\text { D.Olkoski } 14 \text { (ICN162488) }\end{array}$ & $\begin{array}{l}\text { S } 30^{\circ} 08^{\prime} 23.2^{\prime \prime} ; \\
\text { W } 52^{\circ} 01 ' 27.3^{\prime \prime}\end{array}$ & & $\begin{array}{c}9 \\
(106)\end{array}$ & 5.6 \\
\hline 15 & $\begin{array}{l}\text { Brazil, Rio Grande do Sul, BR 116, km295. } \\
\text { D.Olkoski } 15 \text { (ICN162489) }\end{array}$ & $\begin{array}{l}\text { S } 30^{\circ} 04^{\prime} 30.5^{\prime \prime} \\
\text { W } 51^{\circ} 20^{\prime} 27.3^{\prime \prime}\end{array}$ & & $\begin{array}{c}4 \\
(25)\end{array}$ & 8.0 \\
\hline 16 & $\begin{array}{l}\text { Brazil, Rio Grande do Sul, Guaíba. D.Olkoski } 16 \\
\text { (ICN162490) }\end{array}$ & $\begin{array}{l}\mathrm{S} 30^{\circ} 11^{\prime} 04.4^{\prime \prime} \\
\mathrm{W} 51^{\circ} 233^{\prime} 44.0^{\prime \prime}\end{array}$ & & $\begin{array}{c}6 \\
(65)\end{array}$ & 12.6 \\
\hline 17 & $\begin{array}{l}\text { Brazil, Rio Grande do Sul, Barra do Ribeiro. } \\
\text { D.Olkoski } 17 \text { (ICN162491) }\end{array}$ & $\begin{array}{l}\text { S } 30^{\circ} 18^{\prime} 53.6^{\prime \prime} \\
\text { W } 51^{\circ} 25^{\prime} 02.3^{\prime \prime}\end{array}$ & & $\begin{array}{c}6 \\
(50)\end{array}$ & 6.0 \\
\hline 18 & $\begin{array}{l}\text { Brazil, Rio Grande do Sul, Capão da Porteira. } \\
\text { D.Olkoski } 18 \text { (ICN162492) }\end{array}$ & $\begin{array}{l}\mathrm{S} 30^{\circ} 06^{\prime} 10.7^{\prime \prime} \\
\mathrm{W} 50^{\circ} 42^{\prime} 01.8^{\prime}\end{array}$ & & $\begin{array}{c}3 \\
(31)\end{array}$ & 6.4 \\
\hline 19 & $\begin{array}{l}\text { Brazil, Rio Grande do Sul, Capivari do Sul. } \\
\text { D.Olkoski } 19 \text { (ICN162493) }\end{array}$ & $\begin{array}{l}\text { S } 30^{\circ} 07^{\prime} 59.7^{\prime \prime} \\
\text { W } 50^{\circ} 34^{\prime} 38.7^{\prime \prime}\end{array}$ & & $5(41)$ & 14.6 \\
\hline 20 & $\begin{array}{l}\text { Brazil, Rio Grande do Sul, Capivari do Sul. } \\
\text { D.Olkoski } 20 \text { (ICN162494) }\end{array}$ & $\begin{array}{l}\text { S } 30^{\circ} 11^{\prime} 00.4 ” \\
\text { W } 50^{\circ} 30^{\prime} 13.0^{\prime \prime}\end{array}$ & & $7(97)$ & 18.5 \\
\hline 21 & $\begin{array}{l}\text { Brazil, Rio Grande do Sul, Palmares do Sul. } \\
\text { D.Olkoski } 21 \text { (ICN162495) }\end{array}$ & $\begin{array}{l}\text { S } 30^{\circ} 22^{\prime} 11.2^{\prime \prime} \\
\text { W } 50^{\circ} 29^{\prime} 42.3^{\prime \prime}\end{array}$ & & $4(42)$ & 0.0 \\
\hline 22 & $\begin{array}{l}\text { Brazil, Rio Grande do Sul, Bacopari. D.Olkoski } 22 \\
\text { (ICN162496) }\end{array}$ & $\begin{array}{l}\text { S } 30^{\circ} 28^{\prime} 30.3 " ; \\
\text { W } 50^{\circ} 28^{\prime} 07.1^{\prime \prime}\end{array}$ & & $3(33)$ & 3.0 \\
\hline
\end{tabular}

To be continued 
D Olkoski and MT Schifino-Wittmann

Table 1, continuation

\begin{tabular}{|c|c|c|c|c|c|}
\hline Population & Locality and voucher & $\begin{array}{c}\text { Geographical } \\
\text { coordinates }\end{array}$ & $n=13^{a}$ & $2 n=26^{a}$ & $\begin{array}{c}\text { Polysomatic } \\
\text { cells }(\%)^{\mathbf{b}}\end{array}$ \\
\hline 23 & $\begin{array}{l}\text { Brazil, Rio Grande do Sul, RST 101; km94. } \\
\text { D.Olkoski } 23 \text { (ICN162497) }\end{array}$ & $\begin{array}{l}\text { S } 30^{\circ} 26^{\prime} 07.4^{\prime \prime} \\
\text { W050'29'59.1" }\end{array}$ & & $8(78)$ & 16.6 \\
\hline 24 & $\begin{array}{l}\text { Brazil, Rio Grande do Sul, Solidão. D.Olkoski } 24 \\
\text { (ICN162498) }\end{array}$ & $\begin{array}{l}\text { S } 30^{\circ} 44^{\prime} 31.7^{\prime \prime} \\
\text { W } 50^{\circ} 36^{\prime} 35.7^{\prime \prime}\end{array}$ & & 7 (89) & 24.7 \\
\hline 25 & $\begin{array}{l}\text { Brazil, Rio Grande do Sul, RST 101; km131,5. } \\
\text { D.Olkoski } 25 \text { (ICN162499) }\end{array}$ & $\begin{array}{l}\text { S } 30^{\circ} 52^{\prime} 47.0^{\prime \prime} \\
\text { W } 50^{\circ} 42^{\prime} 46.6^{\prime \prime}\end{array}$ & & $4(30)$ & 13.3 \\
\hline 26 & $\begin{array}{l}\text { Brazil, Rio Grande do Sul, Mostardas. D.Olkoski } \\
26 \text { (ICN162500) }\end{array}$ & $\begin{array}{l}\text { S } 30^{\circ} 59^{\prime} 47.0^{\prime \prime} \\
\text { W } 50^{\circ} 49^{\prime} 40.2^{\prime \prime}\end{array}$ & & $3(32)$ & 9.4 \\
\hline 27 & $\begin{array}{l}\text { Brazil, Rio Grande do Sul, Porto Alegre. N. } \\
\text { Dahmer s/n }\end{array}$ & $\begin{array}{l}\text { S } 30^{\circ} 01^{\prime} 37.28^{\prime \prime} ; \\
\text { W } 51^{\circ} 12^{\prime} 02.83^{\prime \prime}\end{array}$ & & $1(15)$ & 13.3 \\
\hline 28 & $\begin{array}{l}\text { Brazil, Rio Grande do Sul, Parobé. D.Olkoski } 27 \\
\text { (ICN162501) }\end{array}$ & $\begin{array}{l}\text { S } 29^{\circ} 38^{\prime} 30.8^{\prime \prime} \\
\text { W } 50^{\circ} 48^{\prime} 28.0^{\prime \prime}\end{array}$ & $3(12)$ & & \\
\hline 29 & $\begin{array}{l}\text { Brazil, Rio Grande do Sul, Rodeio Bonito. } \\
\text { D.Olkoski } 28 \text { (ICN162502) }\end{array}$ & $\begin{array}{l}\text { S } 29^{\circ} 31^{\prime} 06.3^{\prime \prime} \\
\text { W } 50^{\circ} 43^{\prime} 57.9^{\prime \prime}\end{array}$ & $3(24)$ & & \\
\hline 30 & $\begin{array}{l}\text { Brazil, Rio Grande do Sul, Farroupilha. D.Olkoski } \\
29 \text { (ICN162503) }\end{array}$ & $\begin{array}{l}\text { S } 29^{\circ} 24^{\prime} 11.1^{\prime \prime} \\
\text { W } 51^{\circ} 20^{\prime} 58^{\prime \prime}\end{array}$ & $5(37)$ & & \\
\hline 31 & $\begin{array}{l}\text { Brazil, Rio Grande do Sul, Farroupilha. D.Olkoski } \\
30 \text { (ICN162504) }\end{array}$ & $\begin{array}{l}\text { S } 29^{\circ} 28^{\prime} 10.7^{\prime \prime} \\
\text { W } 51^{\circ} 211^{\prime} 11.4^{\prime \prime}\end{array}$ & $3(32)$ & & \\
\hline 32 & $\begin{array}{l}\text { Brazil, Rio Grande do Sul, São Sebastião do Caí. } \\
\text { D.Olkoski } 31 \text { (ICN162505) }\end{array}$ & $\begin{array}{l}\text { S } 29^{\circ} 33^{\prime} 59.4^{\prime \prime} \\
\text { W } 51^{\circ} 21^{\prime} 37.0^{\prime \prime}\end{array}$ & $2(8)$ & & \\
\hline 33 & $\begin{array}{l}\text { Brazil, Rio Grande do Sul, São Sebastião do Caí. } \\
\text { D.Olkoski } 32 \text { (ICN162506) }\end{array}$ & $\begin{array}{l}\text { S 293 } 37^{\prime} 33.6^{\prime \prime} \\
\text { W } 51^{\circ} 18^{\prime} 37.2^{\prime \prime}\end{array}$ & $4(23)$ & & \\
\hline 34 & $\begin{array}{l}\text { Brazil, Rio Grande do Sul, Portão. D.Olkoski } 33 \\
\text { (ICN162507) }\end{array}$ & $\begin{array}{l}\text { S } 29^{\circ} 42^{\prime} 45.9^{\prime \prime} \\
\text { W } 51^{\circ} 10^{\prime} 54.6^{\prime \prime}\end{array}$ & $4(22)$ & & \\
\hline 35 & $\begin{array}{l}\text { Brazil, Rio Grande do Sul, Sapucaia do Sul. } \\
\text { D.Olkoski } 34 \text { (ICN162508) }\end{array}$ & $\begin{array}{l}\text { S } 29^{\circ} 49^{\prime} 13.5^{\prime \prime} \\
\text { W } 51^{\circ} 10^{\prime} 31.0^{\prime \prime}\end{array}$ & $2(13)$ & & \\
\hline 36 & $\begin{array}{l}\text { Brazil, Rio Grande do Sul, Esteio. D.Olkoski } 35 \\
\text { (ICN162509) }\end{array}$ & $\begin{array}{l}\text { S } 29^{\circ} 51^{\prime} 51.9^{\prime \prime} \\
\text { W } 51^{\circ} 10^{\prime} 50.7^{\prime \prime}\end{array}$ & $2(13)$ & & \\
\hline 37 & $\begin{array}{l}\text { Brazil, Rio Grande do Sul, Canoas. D.Olkoski } 36 \\
\text { (ICN162510) }\end{array}$ & $\begin{array}{l}\text { S } 29^{\circ} 52^{\prime} 59.9^{\prime \prime} \\
\text { W } 51^{\circ} 12^{\prime} 39.6^{\prime \prime}\end{array}$ & $\begin{array}{c}10 \\
(46)\end{array}$ & $\begin{array}{c}10 \\
(103)\end{array}$ & 25.8 \\
\hline 38 & $\begin{array}{l}\text { Brazil, Rio Grande do Sul, Xangrilá. D.Olkoski } 37 \\
\text { (ICN162511) }\end{array}$ & $\begin{array}{l}\text { S } 29^{\circ} 46^{\prime} 11.8 " \\
\text { W } 50^{\circ} 03 ' 24.1\end{array}$ & & $2(18)$ & 5.5 \\
\hline 39 & $\begin{array}{l}\text { Brazil, Rio Grande do Sul, Rondinha. D.Olkoski } \\
38 \text { (ICN162512) }\end{array}$ & $\begin{array}{l}\text { S 2929'27.1"'; } \\
\text { W } 49^{\circ} 52^{\prime} 15.1 "\end{array}$ & & $3(32)$ & 6.2 \\
\hline 40 & $\begin{array}{l}\text { Brazil, Rio Grande do Sul, Santo Antonio da } \\
\text { Patrulha. D.Olkoski } 39 \text { (ICN162513) }\end{array}$ & $\begin{array}{l}\text { S } 29^{\circ} 52^{\prime} 19.1 " \\
\text { W } 50^{\circ} 25^{\prime} 11.3 "\end{array}$ & & $2(24)$ & 12.5 \\
\hline 41 & $\begin{array}{l}\text { Brazil, Rio Grande do Sul, Santo Antonio da } \\
\text { Patrulha. D.Olkoski } 40 \text { (ICN162514) }\end{array}$ & $\begin{array}{l}\text { S } 29^{\circ} 52^{\prime} 59.0^{\prime \prime} \\
\text { W050'3 }\end{array}$ & & $3(26)$ & 11.5 \\
\hline 42 & $\begin{array}{l}\text { Brazil, Rio Grande do Sul, Glorinha. D.Olkoski } 41 \\
\text { (ICN162515) }\end{array}$ & 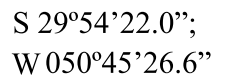 & & $2(24)$ & 12.5 \\
\hline 43 & $\begin{array}{l}\text { Brazil, Rio Grande do Sul, Nova Santa Rita. } \\
\text { D.Olkoski } 42 \text { (ICN162516) }\end{array}$ & $\begin{array}{l}\text { S } 29^{\circ} 50^{\prime} 20.3^{\prime \prime} \\
\text { W } 51^{\circ} 17^{\prime} 54.3^{\prime \prime}\end{array}$ & & $7(67)$ & 6.0 \\
\hline
\end{tabular}

To be continued 
Table 1. continuation

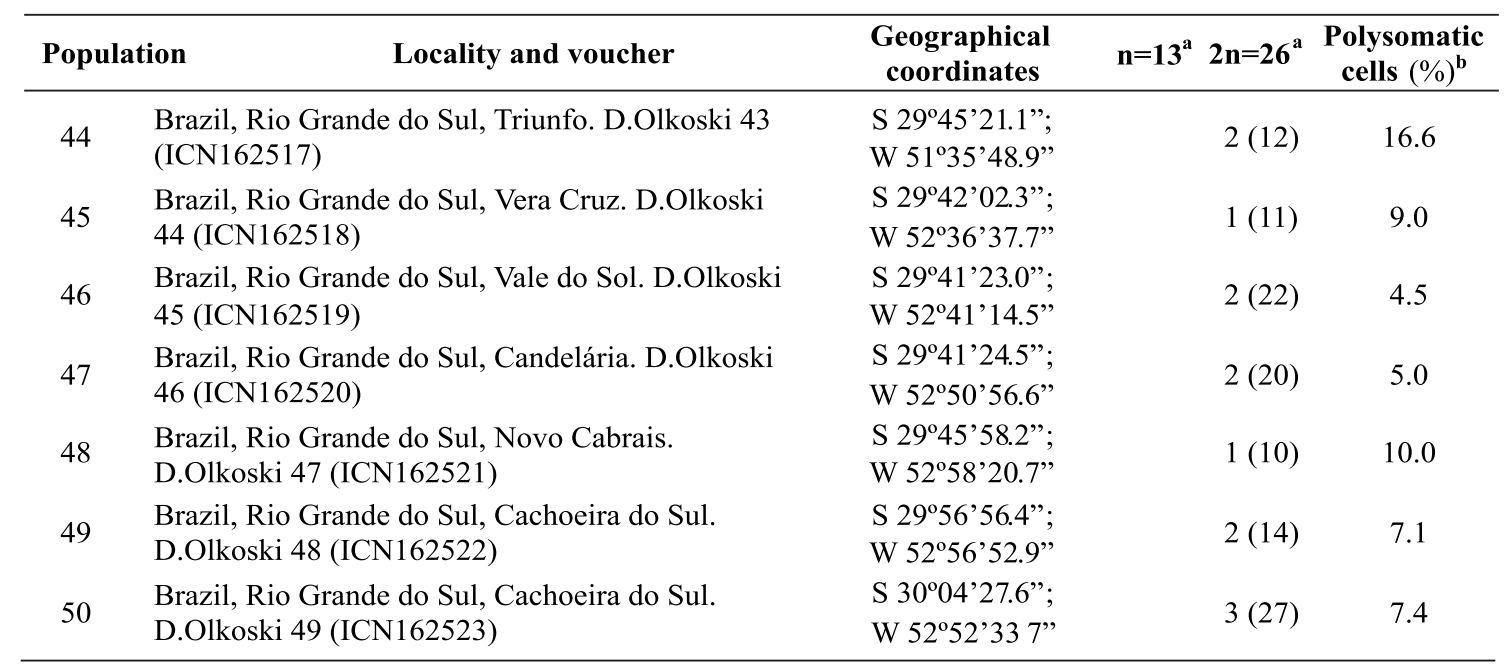

${ }^{a}$ Number of individuals and cells (between brackets) analyzed.

${ }^{\mathrm{b}}$ Percentage of polysomatic cells in seedling root-tips.

(Table 1). Almost all of polysomatic cells were tetraploid (Figure 1d) and a few triploid. Percentage of polysomatic cells (in root-tips with pretreatment) among populations ranged from 3.0 to 28.2 among populations. However polysomaty was absent from the root-tip cells of adult plants as well as in pollen-mother cells.

Witkus and Berger (1947) working with Mimosa pudica $\mathrm{L}$., detected polysomaty in seedling root-tips from 1 to $4 \mathrm{~mm}$ length, but in $10 \mathrm{~mm}$ long root-tips no polysomatic cells were found. Seijo $(1993,1999)$ commented that polysomatic cells up to four times the diploid number of chromosomes were found in root-tips of some Mimosa species. Therefore, the results for M. bimucronata support Witkus and Berger (1947) suggestion that polysomaty in Mimosa pudica L. would be a mechanism for a rapid seedling establishment. On the other hand, absence of polysomatic cells in the germinative line would avoid the potential formation of polyploid gametes (Galbraith et al. 1991).

In M. bimucronata pollen-mother cells are joined two-by-two even before the onset of meiosis and remain linked during all the meiotic division (Figure 2a to 2e) until the formation of pollen grain polyads (Figure 2f). The sporogenic cells undergo mitosis and, the two resulting cells (Figure 2a) remain attached during meiotic division, that is synchronous in both sister cells (Figure $2 b$ to $2 \mathrm{e}$ ). At the end of meiosis the microspores of each set of two sister cells remain linked, originating the polyad, in fact a bitetrad of two groups of four pollen grains, each from one of the sister cells, that are dispersed in that form (Figure 2f).

Seijo and Neffa (2004) described the formation of polyads in M. bimucronata and proposed that the number of pollen grains per polyad is determined by the number of cells that remain attached after the last mitotic divisions of the sporogenic cell, that are two in M. bimucronata. In Acacia Mill., also a Mimosoideae genus, the mechanism of polyad formation was studied in detail (Kenrick and Knox 1979, 1982, Fitzgerald et al. 1993). In Acacia, polyads of eight, 16 and up to 32 pollen grains are formed. Polyads with 12 and 16 pollen grains are found in other Mimosa species. According to Seijo and Neffa (2004), the number of pollen grains per polyad would correlate to the numbers of ovules per ovary and, due to the dimensions of $M$. bimucronata stigma, only one polyad would fit per stigma. The occurrence of polyads in M. bimucronata would be an adaptation to ensure high seed set after a single pollination event (Seijo and Neffa 2004)

Concluding, all the $50 \mathrm{M}$. bimucronata populations analyzed were diploid $(2 n=2 x=26, n=13)$ and no intraspecific variability for chromosome number was detected. Polysomaty occurred in varied degrees but only in seedlings root-tips and was absent in root-tips of established plants as well as in pollen-mother-cells, supporting the theory that, in Mimosa, polysomaty is a way to assure seedling establishment. 

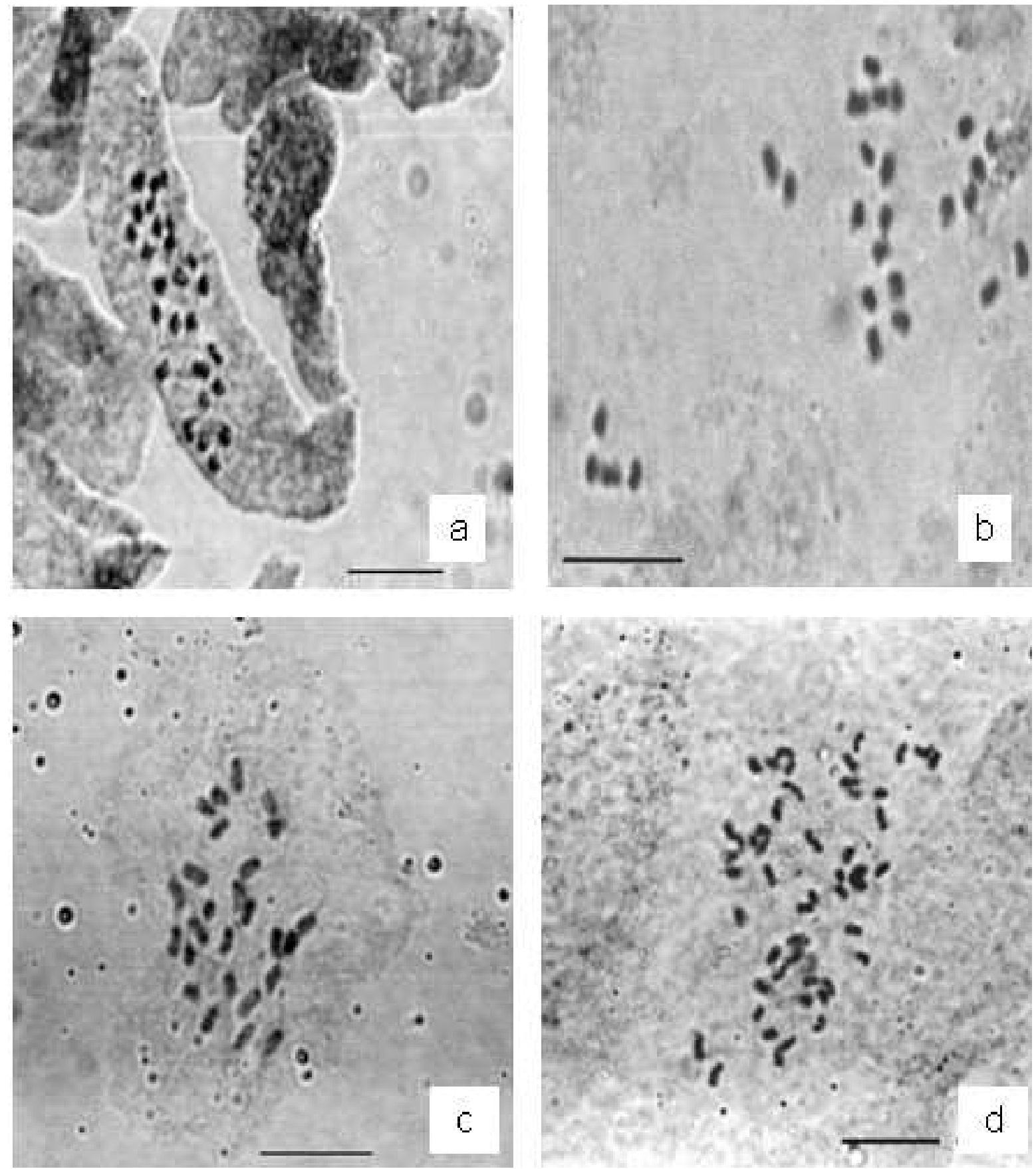

Figure 1. Mitotic metaphases of M. bimucronata (DC.) O. Kuntze. (a) population 19; (b) population 1; (c) population 3, all with 2n = 26; (d) root-tip polysomatic cell, with $2 \mathrm{n}=52$, population 3 . Scale bar $=10 \mu \mathrm{m}$.

During all the meiotic process pollen-mother cells remain joined two-by-two, leading to the formation of polyads with eight pollen grains (two sets of four) each, probably facilitating pollen dispersion and pollination efficiency. 
Cytogenetics of Mimosa bimucronata (DC.) O. Kuntze...
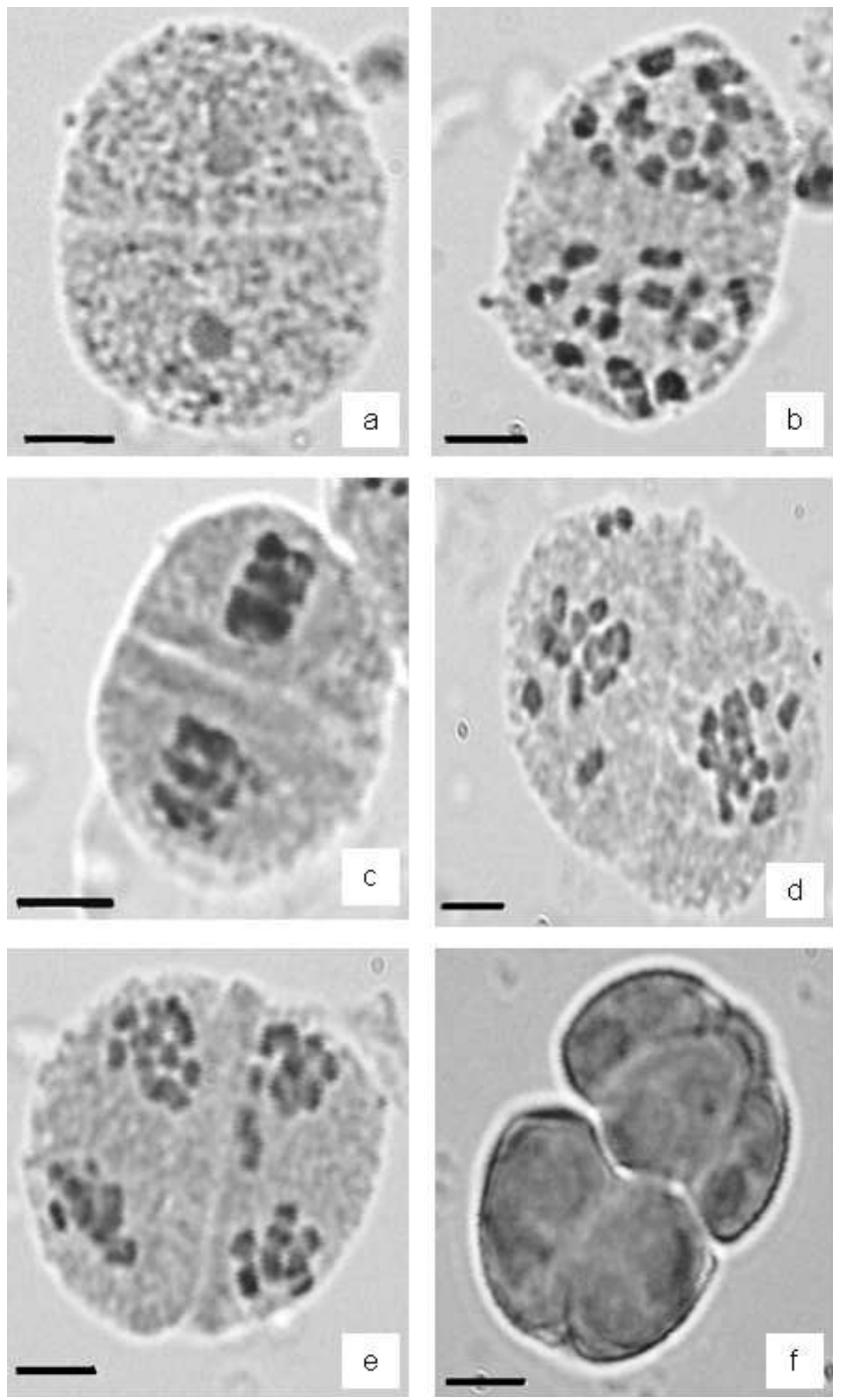

Figure 2. Meiosis in pollen-mother-cells (joined two-by-two) of M. bimucronata (DC.) O. Kuntze (a) interphase; (b) diakinesis showing 13 bivalents ; (c) metaphase I; (d) early anaphase I; (e) late anaphase I; (f) equatorial view of a polyad with eight pollen grains. Scale bar $=10 \mathrm{~mm}$. 


\title{
Citogenética de Mimosa bimucronata (DC.) O. Kuntze (Mimosoideae, Leguminosae): número cromossômico, polissomatia e meiose
}

\begin{abstract}
RESUMO - Os números cromossômicos (somáticos elou gaméticos) foram determinados em plantas oriundas de 50 populations de M. bimucronata (DC.) O. Kuntze coletadas na área de distribuição da espécie no Rio Grande do Sul. Todas as populações analisadas eram diplóides $(2 n=2 x=26, n=13)$. Células polissomáticas (em geral tetraplóides) foram detectadas em ponta de raiz de plântulas em 39 das 41 populações analisadas, variando de 3 a 28,2\% entre populações. mas estavam ausentes nas pontas de raiz de plantas adultas. Polissomatia também estava ausente nas células-mães-de-pólen. Em M. bimucronata as células-mães-depólen estão juntas duas a duas antes do início da meiose, permanecendo unidas durante toda a divisão meiótica até a formação de políades de graõs de pólen. As políades são compostas de dois conjuntos de quatro grãos cada, que são dispersos desta forma o que, de acordo com sugestões prévias, seria uma adaptação para assegurar alta formação de sementes após um único evento de polinização.
\end{abstract}

Palavras-chave: maricá, variabilidade, políades.

\section{REFERENCES}

Alzate-Marin AL, Cervigni GDL, Moreira MA and Barros EG (2005) Seleção assistida por marcadores moleculares visando ao desenvolvimento de plantas resistentes a doenças, com ênfase em feijoeiro e soja. Fitopatologia Brasileira 30: 333 342 .

Anderson JA, Chao S and Liu S (2007) Molecular breeding using a major QTL for fusarium head blight resistance in wheat. Crop Science 47: 112-119.

AOAC. Association of Official Analytical Chemistry (1992) Official methods of Analysis of the Association of Official Analytical Chemistry. $11^{\text {th }}$ ed., AOAC, Washington, 1115p.

Barros LM, Paiva JR, Cavalcanti JJV and Araújo JPP (2002) Cajueiro. In Bruckner $\mathrm{CH}$ (Ed.) Melhoramento de fruteiras tropicais. Editora UFV, Viçosa, p. 159-176.

Broman KW (2001) Review of statistical methods for QTL mapping in experimental crosses. Lab Animal 30: 44-52.

Cavalcanti JJV and Wilkinson MJ (2007) The first genetic maps of cashew (Anacardium occidentale L.). Euphytica 157: 131143.

Crisóstomo JR, Cavalcanti JJV, Barros LM, Alves RE, Freitas JG and Oliveira JN (2002) Melhoramento do cajueiro-anãoprecoce: avaliação da qualidade do pedúnculo e a heterose dos seus híbridos. Revista Brasileira de Fruticultura 24: 477480 .

Alvez MA and Custódio AVC (1989) Citogenética de leguminosas coletadas no estado do Ceará. Revista Brasileira de Genética 12: $81-92$.

Backes P and Irgang B (2002) Árvores do sul. Instituto Souza Cruz, Porto Alegre, 326p.
Barbosa JHC and Faria SM (2006) Aporte de serrapilheira ao solo em estágios sucessionais florestais na Reserva Biológica de Poço das Antas, Rio de Janeiro, Brasil. Rodriguésia 57: 461-476.

Barneby RC (1991) Sensitivae censitae: Mimosa. Memoirs of the New York Botanical Garden 65: 1-835.

Bernaci LC, Goldenberg R and Metzger JP (1998) Estrutura florística de quinze fragmentos florestais ripários na bacia do Jacaré Pepira (SP). Naturalia 23: 23-54.

Bitencourt F, Zocche JJ, Costa S, Souza PZ and Mendes AR (2007) Nucleação de Mimosa bimucronata (DC.) O. Kuntze em áreas degradadas pela mineração de carvão. Revista Brasileira de Biociências 5: 750-752.

Burkart A (1979) Flora ilustrada catarinense. Leguminosasmimosoideas. Herbário Barbosa Rodrigues, Itajaí, 299p.

Dahmer N, Kaminsky P and Schifino-Wittmann MT (2009) Chromosome number and karyotype of the endangered Amazonian woody species "pau-rainha" (Centrolobium paraense Tul.). Crop Breeding and Applied Biotechnology 9: $382-385$.

Federov An A (1969) Chromosome numbers of flowering plants. Academy of Sciences of the USSR, Leningrad, 926p.

Fitzgerald MA, Calder DM and Knox RB (1993) Character state of development and initiation of cohesion between compound pollen grains of Acacia paradoxa. Annals of Botany 71: 51-59.

Galbraith DW, Harkins KR and Knapp S (1991) Systemic endopolyploidy in Arabidopsis thaliana. Plant Physiology 96: 985-989.

Goldblatt P (1981) Cytology and the phylogeny of Leguminosae. In Polhill RM and RavenPM (ed.) Advances in legume systematics 2. Royal Botanic Gardens, Kew, p. 427-463.

Crop Breeding and Applied Biotechnology 11: 27-35, 2011 
Goldblatt P and Johnson DE (2009) Index to plant chromosome numbers. Available at http://mobot.mobot.org/W3T/Search/ ipcn.html. Assessed on Jan 10, 2010.

Kenrick J and Knox RB (1979) Pollen development and cytochemistry in some Australian species of Acacia. Australian Journal of Botany 27: 413-427.

Kenrick J and Knox RB (1982) Function of the polyad in the reproduction of Acacia. Annals of Botany 50: 721-727.

Lorenzi H (1998) Árvores brasileiras: manual de identificação e cultivo de plantas arbóreas nativas do Brasil. Vol. II, Plantarum, Nova Odessa, 368p.

Marchiori JNC (1993) Anatomia da madeira e casca do maricá, Mimosa bimucronata (DC.) O. Kuntze. Ciência Florestal 3 : 85-106.

Morales M, Wulff AF, Fortunato RH and Poggio L (2010) Chromosome and morphological studies in the Mimosa debilis complex (Mimosoideae, Leguminosae) from southern South American. Australian Journal of Botany 58: 12-22.

Nascimento CES, Rodal MJN and Cavalcanti AC (2003) Phytosociology of the remaining xerophytic woodland associated to an environmental gradient at the banks of the São Francisco river - Petrolina, Pernambuco, Brazil. Revista Brasileira de Botânica 26: 271-287.
Reitz R, Klein RM and Reis A (1983) Projeto madeira do Rio Grande do Sul. Selowiana 34/35: $1-525$.

Seijo G (1993) Números cromosómicos en especies argentinas del género Mimosa (Leguminosae). Boletin Sociedad Argentina de Botánica 29: 219-223.

Seijo G (1999) Chromosome studies in Argentinian species of Mimosa. Cytologia 64: 241-246.

Seijo G (2000) Números cromosómicos en especies de Mimosa de Paraguay. Bonplandia 10: 163-167.

Seijo G and Fernández A (2001) Chromosome numbers of some southernmost species of Mimosa L. (Leguminosae). Cytologia 66: $19-23$.

Seijo G and Neffa VGS (2004) The cytological origin of the polyads and their significance in the reproductive biology of Mimosa bimucronata. Botanical Journal of the Linnean Society 144: 343-349.

Witkus ER and Berger CA (1947) Polyploid mitosis in the normal development of Mimosa pudica. Bulletin of the Torrey Botanical Club 74: 179-182. 